ARTICLE

DOI: $10.1038 / s 41467-018-04002-4$

\title{
Distinct mutational signatures characterize concurrent loss of polymerase proofreading and mismatch repair
}

\author{
N.J. Haradhvala1,2, J. Kim², Y.E. Maruvka², P. Polak (1) 1,2,3, D. Rosebrock², D. Livitz (1) 2, J.M. Hess², \\ I. Leshchiner (10 2, A. Kamburov 1,2,3, K.W. Mouw ${ }^{3,4}$, M.S. Lawrence ${ }^{1,2,3}$ \& G. Getz (1) 1,2,3
}

Fidelity of DNA replication is maintained using polymerase proofreading and the mismatch repair pathway. Tumors with loss of function of either mechanism have elevated mutation rates with characteristic mutational signatures. Here we report that tumors with concurrent loss of both polymerase proofreading and mismatch repair function have mutational patterns that are not a simple sum of the signatures of the individual alterations, but correspond to distinct, previously unexplained signatures: COSMIC database signatures 14 and 20. We then demonstrate that in all five cases in which the chronological order of events could be determined, polymerase epsilon proofreading alterations precede the defect in mismatch repair. Overall, we illustrate that multiple distinct mutational signatures can result from different combinations of a smaller number of mutational processes (of either damage or repair), which can influence the interpretation and discovery of mutational signatures.

\footnotetext{
${ }^{1}$ Department of Pathology and Cancer Center, Massachusetts General Hospital, 55 Fruit Street, Boston, MA 02114, USA. ${ }^{2}$ Broad Institute of Harvard and MIT, 415 Main Street, Cambridge, MA 02142, USA. ${ }^{3}$ Harvard Medical School, 25 Shattuck Street, Boston, MA 02115, USA. ${ }^{4}$ Department of Radiation Oncology, Brigham and Women's Hospital, Dana-Farber Cancer Institute, 450 Brookline Ave, HIM 350, Boston, MA 02215, USA. These authors contributed equally: Haradhvala NJ, Kim J, Maruvka YE. These authors jointly supervised this work: Mouw KW, Lawrence MS, Getz G. Correspondence and requests for materials should be addressed to G.G. (email: gadgetz@broadinstitute.org)
} 
C ells have evolved multiple mechanisms to ensure that DNA replication occurs in a timely and accurate manner. However, DNA replication fidelity is frequently compromised in cancer, leading to accumulation of somatic mutations throughout the evolution of the tumor. These mutations serve as a footprint of the tumor's underlying DNA damage and repair landscape $e^{1,2}$. Specific mutational signatures have been identified in tumors with mutations in the exonuclease (proofreading) domain of polymerase epsilon (POLE), as well as tumors with mutations or epigenetic silencing of genes in the mismatch repair pathway $(M M R)^{3,4}$. Until recently, it was thought that simultaneous loss of both POLE or polymerase delta (POLD1) proofreading and MMR function could not be tolerated by cells due to excessive accumulation of mutations ${ }^{5-7}$. However, a recent analysis of tumors from children with biallelic germline MMR deficiency (bMMRD) revealed a subset of tumors with remarkably high mutation burdens ( $>250$ mutations $/ \mathrm{Mb}$ ) that also had a somatic mutation in $P O L E$ or $P O L D 1^{8}$. In a follow-up study, concurrent to our own, the mutational spectra observed in a large number of adult tumors were clustered, revealing that a subset of endometrial and colorectal cancers have spectra similar to the pediatric bMMRD POLE-mutated cases ${ }^{9}$. In addition, a study of more than 500 endometrial tumors revealed that nearly half (12/ 30 ) of the tumors with a POLE exonuclease domain mutation also displayed microsatellite instability $(\mathrm{MSI})^{10}$, the characteristic patterns of insertions and deletions associated with loss of MMR function. Here, we investigate the mutational properties of tumors with $P O L E / P O L D 1$ proofreading loss and MSI. We identify mutational signatures associated with concurrent $P O L E$ or POLD1 exonuclease mutations (POLE-exo* or POLD1-exo*) and MMR loss that are distinct from the signatures associated with either event in isolation.

\section{Results}

Proofreading and MMR deficiency in endometrial cancer. To characterize the relationship among POLE/POLD1 proofreading, MMR function, and mutational signatures, we first sought to identify tumors with simultaneous loss of both pathways. Endometrial tumors are known to possess relatively high rates of both events, so we began our search in The Cancer Genome Atlas

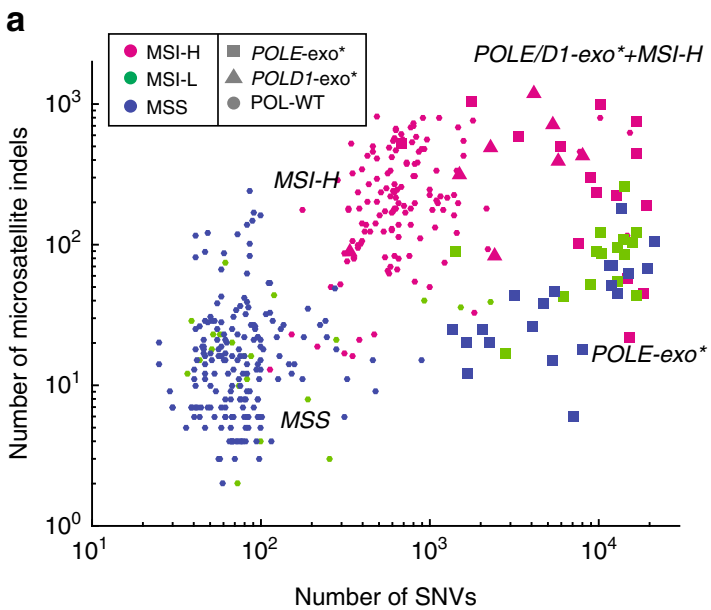

(TCGA) cohort of 531 endometrial (UCEC) samples ${ }^{3}$. Experimental MSI status (MSI-high [MSI-H], MSI-low [MSI-L], or microsatellite stable (MSS)) was available for 410 tumors ${ }^{11}$, and we identified 15 samples classified as MSI-H that also harbored a somatic point mutation in the POLE exonuclease domain (codons 268-471 12 ), as well as eight MSI-H samples with no POLE proofreading mutation but instead possessing a POLD1 exonuclease domain mutation (codons $304-517^{12}$, Supplementary Data 1 and 2). The majority of these 23 tumors displayed large numbers of microsatellite insertions and deletions (MS indels) indicative of MMR loss ${ }^{13}$ as well as a high point mutation burden consistent with loss of polymerase proofreading (Fig. 1a). Many of these cases (7/15 POLE-mutated MSI-H tumors and 5/8 POLD1-mutated MSI-H tumors) were found to have epigenetic silencing of $M L H 1$, the most common mechanism that causes loss of MMR in tumors, suggesting that the high MS indel rates observed in these tumors were indeed due to MMR deficiency. The majority (7/8) of POLE and all (3/3) POLD1-mutated tumors classified as MSI-H but lacking MLH1 silencing had either a truncating/frame shift mutation or deletion of one of the four major MMR pathway genes (MLH1, MSH2, MSH6, or PMS2), although due to the high mutation rate of POLE, many tumors may have such mutations in MMR genes by chance.

Tumors with either of the two most common POLE exonuclease domain mutations (P286R or V411L) were rarely MSI-H (2/30 cases), whereas the majority of tumors with any other POLE-exo mutation were MSI-H (13/20) (Fisher's exact test $p=1.6 \times 10^{-5}$; Fig. 1b). Interestingly, POLE-exo* tumors with a V411L mutation were often classified as MSI-L (7/12 cases) despite having MS indel burdens that resembled MSI-H samples with a wild-type (WT) POLE/POLD1 exonuclease domain. In contrast, most MSI-L tumors with either a P286R POLE mutation or with WT exonuclease domain did not have high numbers of MS indels (Fig. 1b). Therefore, whereas P286R and WT POLE samples were likely classified as MSI-L due to chance mutation of a locus assayed in the Bethesda test, the V411L POLE samples might actually represent bona fide MMR-deficient (i.e., MSI-H) cases that were misclassified as MSI-L by the Bethesda assay, which was developed and validated in colon tumor cohorts (in which concurrent POLE/POLD1 proofreading loss and MSI has not been observed).

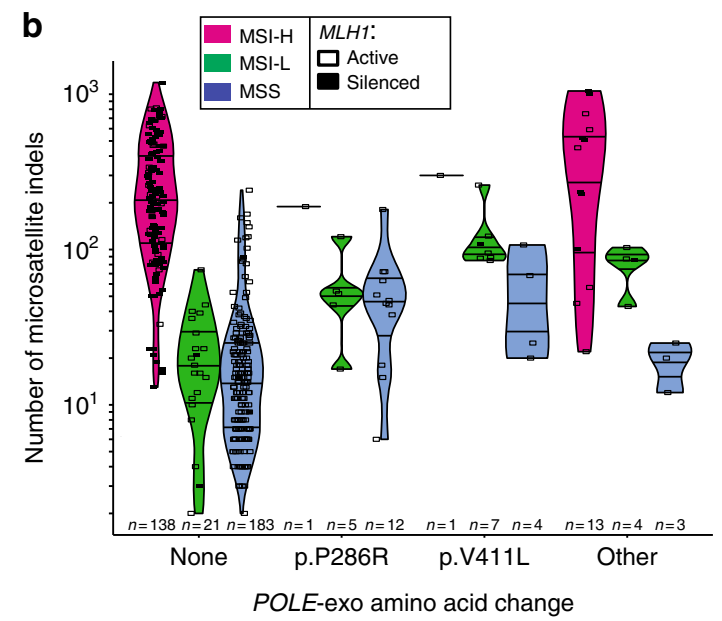

Fig. 1 The landscape of single-nucleotide variants (SNVs) and microsatellite indels in endometrial cancer. a Number of SNVs and microsatellite indels for tumors in the TCGA endometrial cohort (UCEC). Colors represent the TCGA microsatellite classification determined using the Bethesda protocol, ${ }^{11}$ and shapes represent the mutation status of POLE and POLD1 exonuclease domains. All samples with available Bethesda classification and MS indel calls are shown $(n=401)$. b Number of microsatellite indels for endometrial tumors with different POLE-exo* mutations. Color represents MSI status, and tumors with MLH1 epigenetic silencing are displayed as filled boxes. P286R and V411L are the two most common POLE exonuclease domain mutations in the cohort. All samples with Bethesda classification, MS indel calls, and MLH1 silencing calls available are shown ( $n=392)$ 
We next aimed to determine whether POLE/POLD1- and MSImediated mutagenesis occurred simultaneously in the same cancer cells, or whether the events were separated spatially (i.e., occurring in distinct subclones) or temporally (i.e., defective in non-overlapping time intervals). First, we noted that POLE/ POLD1 exonuclease domain mutations were frequently clonal (13/15 POLE and 8/10 POLD1 mutations had an estimated cancer cell fraction $[\mathrm{CCF}]>0.9)$ and that non-clonal $P O L E / P O L D 1$ mutations appeared to be non-functional passenger events (Supplementary Figure 1). This suggests that the POLE/POLD1 proofreading defect was present in all cancer cells, and therefore, present in the same cells as the MMR deficiency.

Mutational signatures of replicative repair deficiency. We next reasoned that if the MMR and polymerase proofreading deficiencies were not present simultaneously, then the joint mutational signature should appear as an additive combination of the signatures corresponding to the individual defects. Alternatively, if the processes were active simultaneously-that is, if the cells had loss of both polymerase proofreading and mismatch repair at the same time-they could potentially yield a distinct mutational signature reflecting the effect of combined loss. To test this hypothesis, we applied our SignatureAnalyzer tool $^{14,15}$ that discovers mutational signatures by applying a Bayesian variant of non-negative matrix factorization ${ }^{14,16}$ to mutation data from a collection of tumors (Methods section).

SignatureAnalyzer identified 12 mutational signatures in the set of 531 endometrial samples (Supplementary Figures 2, 3; Supplementary Data 1, 3; Methods section). To explore these 12 signatures, we correlated their activities (i.e., the estimated number of mutations contributed by each signature to the total mutational burden of the tumor) to the MSI status and POLE/POLD1 exonuclease domain mutation status of each tumor in the cohort. Eight of the 12 signatures were associated with deficiencies in MMR and/or POLE/POLD1 proofreading (4/12 showed no such enrichment; Supplementary Figure 4). Cases with a POLE exonuclease mutation had enrichment of one of three signatures (Fig. 2a, b), designated as signatures E1-E3. Signatures E1 and E2 closely resemble the signature previously associated with somatic POLE exonuclease mutations (COSMIC a

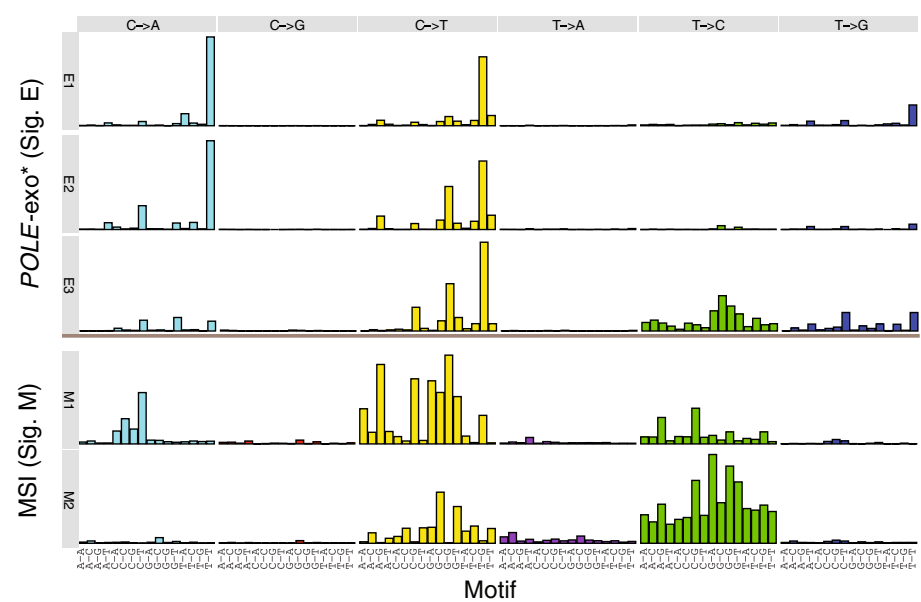

C

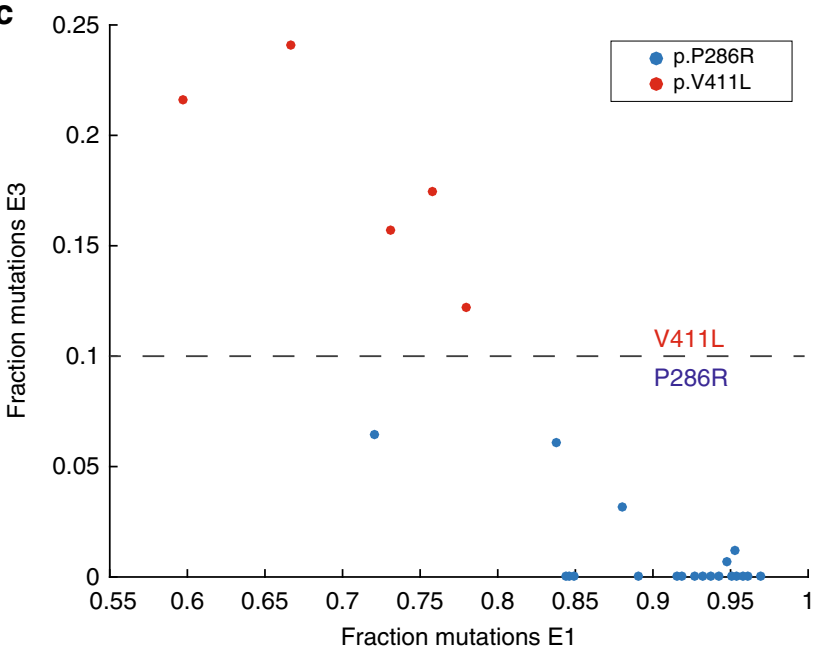

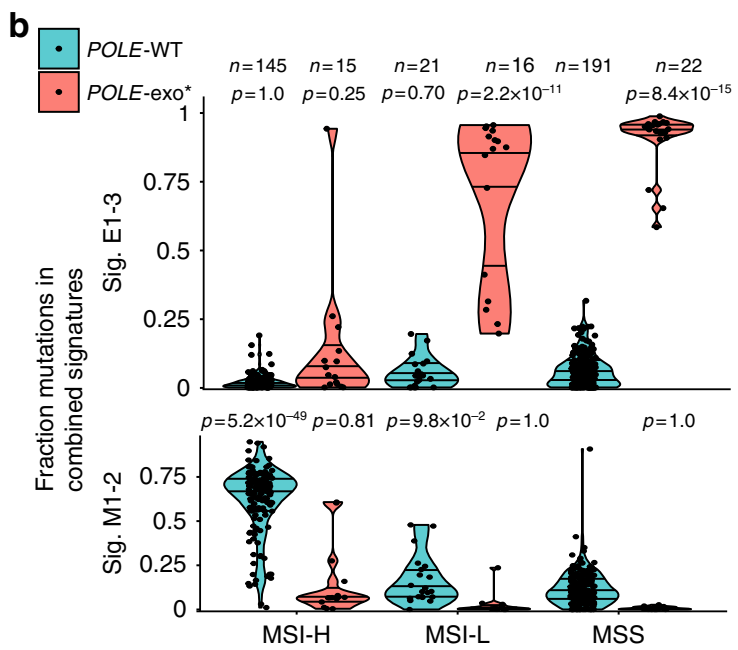

Fig. 2 Identification of mutational signatures associated with individual loss of POLE proofreading or MMR. a SignatureAnalyzer yielded five mutational signatures associated with loss of polymerase proofreading (E1, E2, and E3) or mismatch repair (M1 and $M 2)$. For each mutational signature, the number of mutations is plotted for the 96 trinucleotide mutational contexts. b The fraction of mutations assigned to each signature across patients, segregated by POLE-exo mutations and Bethesda microsatellite classification. The distribution of each group is compared to that of polymerase wild-type MSS samples using a one-tailed rank-sum test. Horizontal lines within each group represent quartile values. c Relative contribution of E1 and E3 signatures to 5 V411L and 20 P286R samples with spectra dominated ( $>75 \%$ contributions) by signatures E1-3. Tumors with the P286R hotspot mutation have primarily contributions from E1, whereas tumors with a V411L hotspot mutation have additional contributions from E3 
signature 10; Supplementary Figure 5$)^{1}$, whereas signature E3 was associated with the POLE V411L hotspot mutation (Fig. 2c). Similarly, we identified two signatures, designated as signatures M1 and M2, characteristic of MSI-H tumors (Fig. 2a, b). These signatures correspond to COSMIC database signatures 6 and 26, respectively, and have previously been noted in MSI samples (Supplementary Figure 5) ${ }^{1}$.

Signatures of concurrent proofreading and MMR deficiency. We next turned our attention to the 15 samples with paired $P O L E$ exonuclease domain mutation and mismatch repair deficiency (POLE-MSI). Despite the presence of both defects in these samples, we observed minimal contributions from signatures E1-3 and M1-2 (Fig. 2b, signatures E1-3 contributed $<20 \%$ mutations in $12 / 15$ samples, and signatures M1-2 $<20 \%$ in $12 / 15$ samples). Instead, two distinct signatures-signatures $\mathrm{C} 1$ and $\mathrm{C} 2-$-were highly enriched in these POLE-MSI tumors (Fig. 3a, b). Signatures $\mathrm{C} 1$ and $\mathrm{C} 2$ shared certain features with the individual MSI (M1-M2) and POLE (E1-E3) signatures but also had distinct motifs such as a unique $\mathrm{G}[\mathrm{C}>\mathrm{A}] \mathrm{T}$ peak (Fig. $3 \mathrm{c}$ and Supplementary Figure 6). These unique motif features may represent mutations that can be repaired by either POLE proofreading or MMR and thus only accumulate in tumors lacking both repair functions (Fig. 3d).

Comparing the $\mathrm{C} 1$ and $\mathrm{C} 2$ signatures to the COSMIC signature database, we found that, when summed using coefficients fit to maximize the cosine similarity, signatures $\mathrm{C} 1$ and $\mathrm{C} 2$ closely resembled a signature of unknown etiology, COSMIC signature 14 (Fig. 3e), more than expected by chance (Supplementary Figure $7 \mathrm{a}, \mathrm{b}$ and Supplementary Data 4$)$. Although the activity of signatures $\mathrm{C} 1$ and $\mathrm{C} 2$ were correlated in our analysis $\left(R^{2}=0.26\right)$, some tumors exhibited high activity of signature $\mathrm{C} 1$ relative to signature C2 (Supplementary Figure 8), demonstrating that indeed two distinct signatures are active in these samples.

Finally, in addition to identifying signatures of concurrent POLE-exo* and MMR deficiency, we found an additional signature (designated as signature D1) that was highly active ( $>1000$ mutations and $>30 \%$ of mutation burden) in $4 / 10 \mathrm{MSI}-\mathrm{H}$ tumors that also had a mutation in the exonuclease domain of POLD1 (Fig. 4a, b). This signature closely resembled COSMIC signature 20 (Fig. 4a and Supplementary Figure 5), which had been associated with MMR deficiency but had not been previously linked to $P O L D 1$ proofreading deficiency. In the four tumors with a POLD1 mutation and high levels of signature D1, the mutation was located within the exonuclease domain (Fig. 4c, d), including residues D316 and E318 within the exonuclease active site. Mutations at the homologous position of D316 in yeast had been shown to impair exonuclease activity ${ }^{17,18}$, and a D316N mutation was identified in an ultramutated gastic tumor ${ }^{19}$. An S478N mutation was also associated with high signature D1 activity, and the same mutation in the germline has been associated with increased risk of early-onset colorectal cancer ${ }^{20}$. In one case with high signature D1 activity, there was no exonuclease mutation, but the tumor did have an L606M mutation in the POLD1 polymerase domain (Fig. 4c, d), which had been shown in vitro to increase polymerase delta error rate ${ }^{21}$ and had also been observed in hypermutated glioblastomas with germline biallelic mismatch repair deficiency ${ }^{8,9}$. In the remaining six POLD1-exo* samples with MSI-H, two also had a POLE-exo mutation and strong contributions from signatures $\mathrm{C} 1$ and $\mathrm{C} 2$, making it difficult to conclude whether the mutations do not impact POLD1 exonuclease function or are overwhelmed by the a
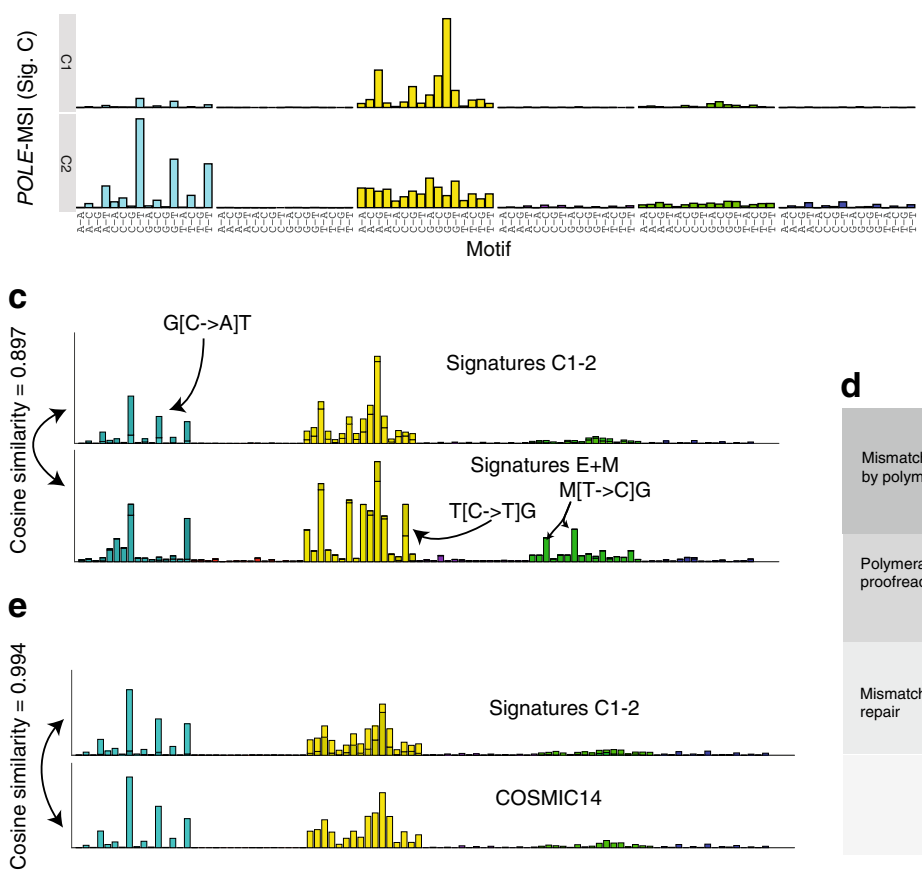

b

d
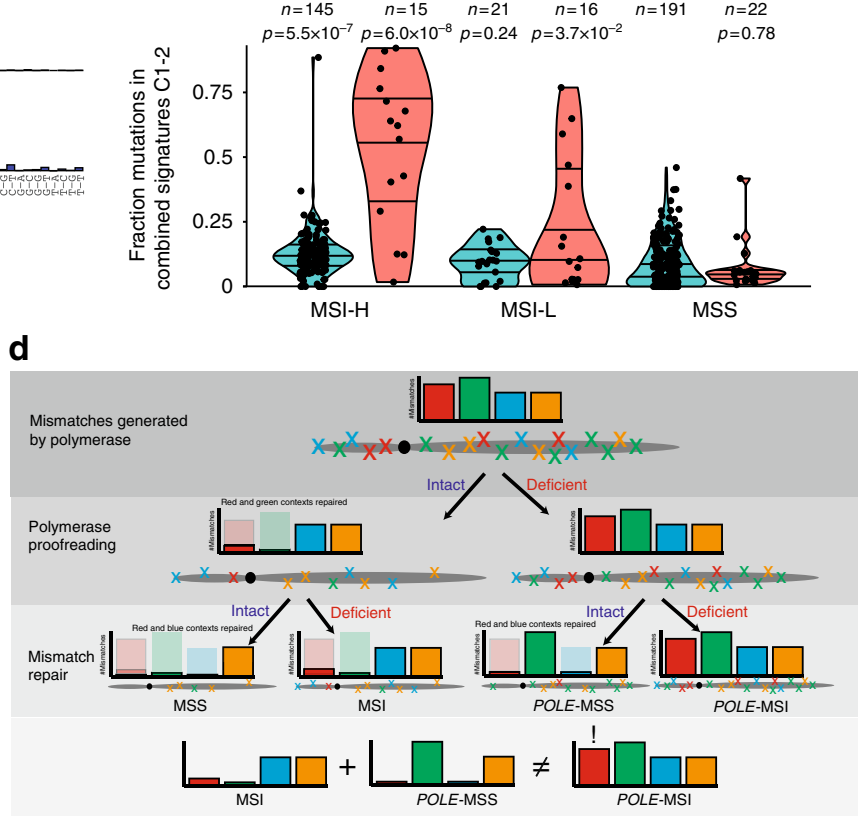

Fig. 3 Identification of mutational signatures associated with paired loss of POLE proofreading and MMR. a SignatureAnalyzer identified two mutational signatures associated with paired POLE-exo mutations and MSI (C1 and C2). $\mathbf{b}$ The fraction of mutations across all cases assigned to signatures $\mathrm{C} 1$ and $\mathrm{C} 2$, segregated by POLE-exo* and MSI status. The distribution of each group is compared to that of polymerase wild-type MSS samples using a one-tailed ranksum test. Horizontal lines within each group represent quartile values. c The five signatures of individual repair defects (signatures M1, M2, E1, E2, and E3) were combined to maximize cosine similarity to a combination of the two signatures of paired defects ( $\mathrm{C} 1$ and $\mathrm{C} 2$ ). Each set of signatures retains several unique peaks, highlighted with arrows. $\mathrm{M}$ represents either $\mathrm{A}$ or $\mathrm{C}$ nucleotides. $\mathbf{d}$ Model highlighting the non-additive mutational signature contributions of combined mutational processes. e A linear combination of the signatures C1 and C2 closely resembles COSMIC signature 14 
a

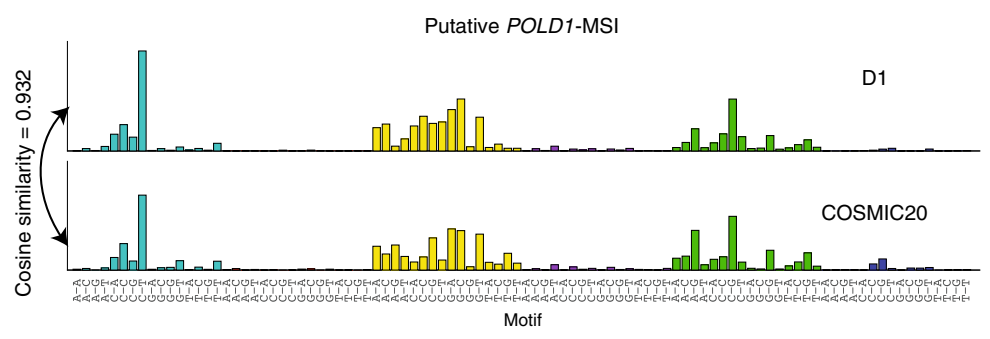

Moti

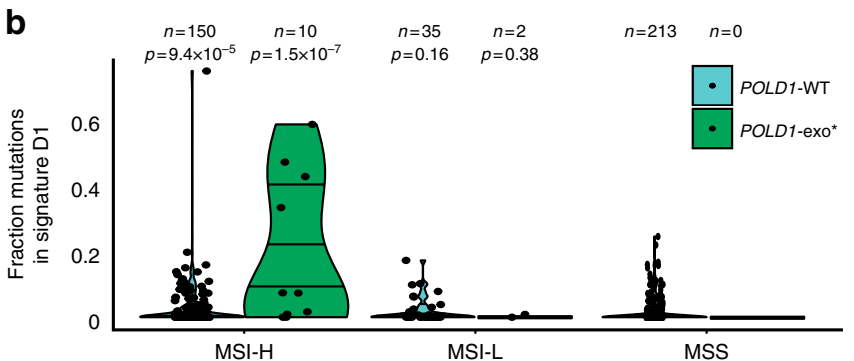

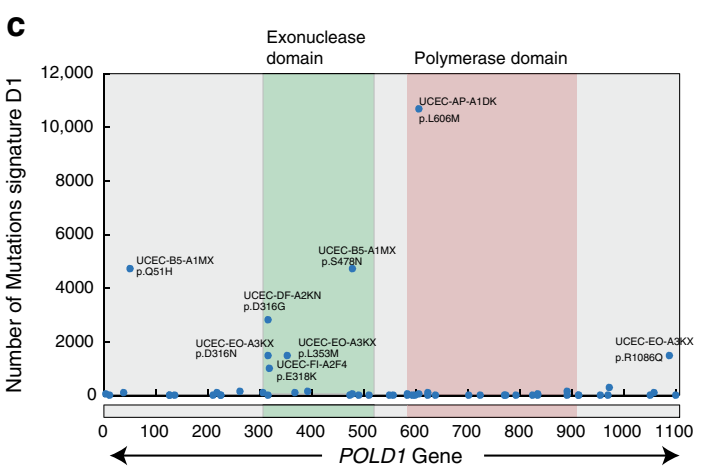

d

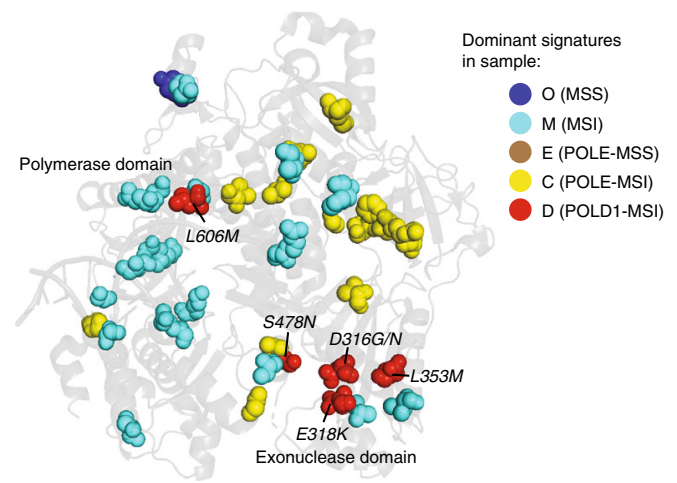

Fig. 4 A distinct mutational signature is associated with paired loss of POLD1 proofreading and MMR. a The 96 trinucleotide mutational spectrum of signature D1 and comparison to COSMIC signature 20. b The fraction of mutations assigned to signature D1 across the cohort, segregated by POLD1-exo* and MSI status. The distribution of each group is compared to that of polymerase wild-type MSS samples using a one-tailed rank-sum test. c Association of signature D1 with mutations in POLD1. d Location of POLD1 mutations mapped to the corresponding location on the yeast POLD1 homolog (PDB ID: 3IAY). Amino acids are color coded by the dominant mutational signature in the sample
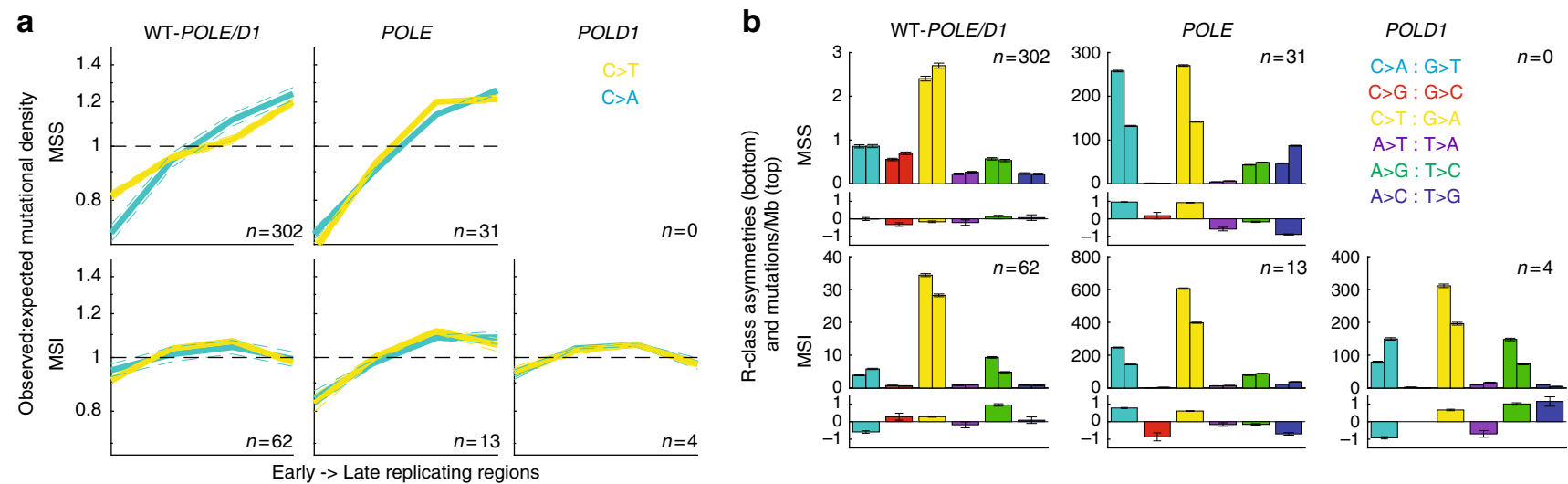

Fig. 5 Genomic properties of repair deficiency. a Replication timing profiles of tumors with all combinations of polymerase and MMR deficiencies. Subsets of tumors with spectra dominated by the signature corresponding to each defect were selected (Supplementary Data 6). No POLD1-MSS samples were present in this data set. The $y$-axis shows enrichment in mutational density in one of four replication timing quartiles normalized by the expected density, assuming a flat background mutation rate. Only the two most frequent substitutions across all signatures, $C>T$ and $C>A$, are shown. $\mathbf{b}$ Replication strand (R-class) mutational asymmetries. The top panel of each subplot shows stranded mutational densities for each base pair change. In each set of twin bars, the left bar represents the rate of mutations where the $A$ or $C$ of the $A: T$ or $C: G$ base pair is predicted to be on the leading strand template, and the right bar where the $A$ or $C$ is on the lagging-strand template. In the lower panel, the $\log _{2}$ ratio of these bars is shown, representing the asymmetry between the leading and lagging strands for this substitution type

effect of the POLE-exo mutation. However, the remaining 4/ 6 samples displayed typical MSI spectra dominated by signatures M1 and M2, suggesting their respective POLD1 alterations (R306C, V392M, V477M, and V489A) do not impact exonuclease function. Notably all eight signatures described above can be clearly seen in the mutation spectrum of each individual patient with the corresponding defect (Supplementary Figure 9).
To extend and validate our findings, we expanded our analysis beyond endometrial cancer. First, we examined somatic mutation data from ten $P O L E / P O L D 1$-mutated glioblastoma tumors ${ }^{8}$ (seven with POLE-exo* and three with POLD1-exo*) from patients with bMMRD. We combined these cases with 61 TCGA endometrial cases that had either a POLE or POLD1 proofreading mutation, including both MSI and MSS cases, and reanalyzed the 
mutational signatures. This analysis rediscovered signatures $\mathrm{C}$, $\mathrm{C} 2$, and $\mathrm{D}$ and found that both signatures $\mathrm{C} 1$ and $\mathrm{C} 2$ were exclusive to the seven bMMRD samples with $P O L E$-exo*, and signature D to the three samples with POLD1-exo* (Supplementary Figure 10,11). Next, we expanded our search to tumors from other TCGA cohorts and identified signatures $\mathrm{C} 1$ and $\mathrm{C} 2$ in one stomach tumor (1/400), one pancreatic tumor (1/166), and a single low-grade glioma (1/516) (Supplementary Figure 12). Indeed, all three of these cases had a POLE-exo* mutation and $>10,000$ somatic exome mutations. The stomach sample displayed MLH1 methylation and was MSI-H. MSI status was not available for the other two samples; however, both the stomach and glioma tumors had high levels of MS indels (296 and 98, respectively), indicative of $\mathrm{MMR}$ deficiency ${ }^{13}$ (Supplementary Data 5). Unfortunately, the number of MS indels could not be determined for the pancreatic tumor due to low purity. Thus, the $P O L E$-MSI signature is not exclusive to endometrial tumors, although the frequency is much reduced in non-endometrial $(<1 \%$ of samples) compared to endometrial cancers (19/ 531 samples, $\sim 3.6 \%$ ).

Genomic properties and covariates. To further investigate the mechanistic underpinnings of the putative POLE/POLD1-MSI signatures, we examined the correlation of DNA replication timing with mutational densities in tumors enriched with each signature (Methods section). Although most tumors exhibit increased mutation frequency in late-replicating regions ${ }^{22,23}$, the mutational profiles of MMR-deficient tumors do not show such a correlation ${ }^{2}$, suggesting that the presence of intact mismatch repair is responsible for this variability. The relationship between late replication and increased mutational density has been shown to be particularly strong in $P O L E$-mutated tumors ${ }^{2}$. We observed similar trends here: (i) the genomes of MSS tumors with a POLE proofreading mutation showed increased mutational burden in late-replicating regions ( $20 \%$ increase from mean; Fig. $5 \mathrm{a})$; and (ii) there was no significant correlation with replication timing in MSI tumors with WT POLE exonuclease domain $(p=0.21$ and 0.42 for F-test on slope of $\mathrm{C}>\mathrm{A}$ and $\mathrm{C}>\mathrm{T}$ profiles; Fig. 5a). However, genomes from POLE-MSI tumors did show an enrichment of mutations in later-replicating regions, even in tumors with negligible contributions from signature E (Supplementary Figure 13), suggesting that MMR alone is not the sole contributor to replication-associated variation (Fig. 5a). POLD1exo $^{*}$ genomes did not show the same enrichment (F-test $p=0.49$ and 0.55; Fig. 5a), perhaps indicating that POLE-related errors are unusually biased toward late-replicating regions.

We next characterized replicative (R-class) strand asymmetries. It was previously observed that loss of either MMR or POLE proofreading resulted in replication strand-biased patterns of mutations ${ }^{19,24}$, suggesting that asymmetric patterns of mismatches introduced during leading- vs. lagging-strand synthesis are only balanced when both POLE and MMR mechanisms are functional. These trends were confirmed in this data set, as both POLE-MSI and POLD1-MSI tumors exhibited strong R-class strand bias (Fig. 5b). Examining the replication asymmetries in various contexts, we noted that the asymmetry profiles of POLEMSI genomes most closely resembled those of POLE-MSS genomes, consistent with error-prone mutant POLE contributing the majority of mutations on the leading strand template. On the other hand, the profiles of POLD1-MSI genomes were more similar to those of MSI tumors (with WT POLD1), perhaps suggesting the mutations unveiled by faulty MMR in the presence of functional polymerase proofreading are more heavily a

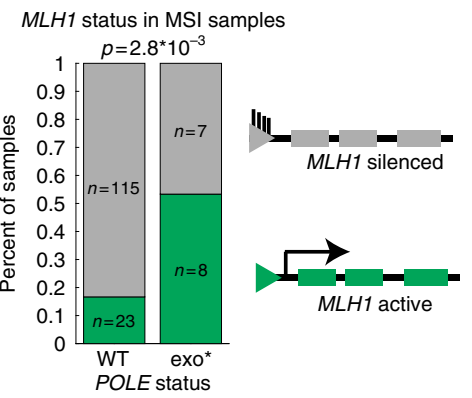

b

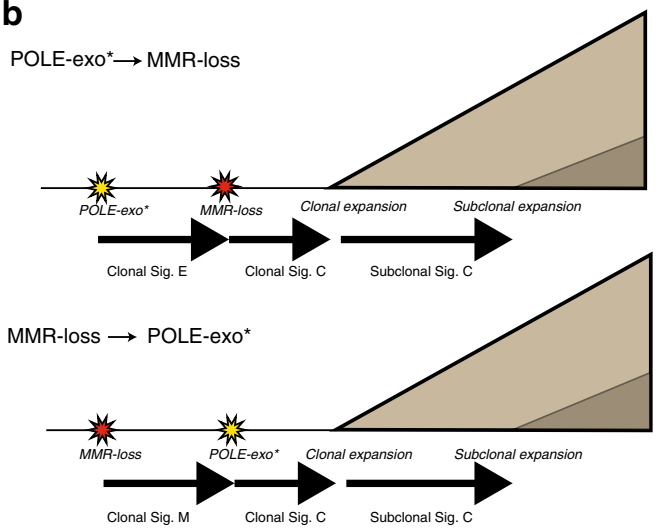

C

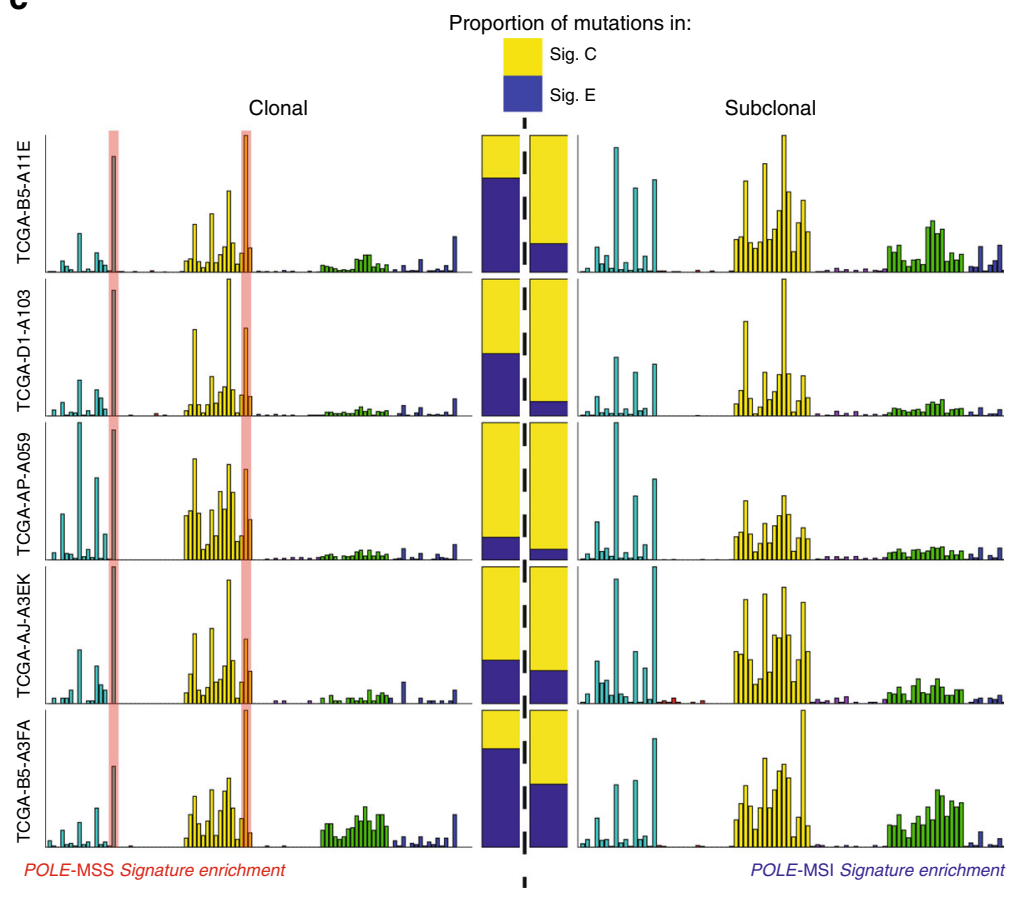

Fig. 6 Timing of POLE-exo* and MSI events. a Frequency of MLH1 silencing as the mechanism of MMR deficiency in POLE WT vs. POLE-exo* cases. $153 / 160$ MSI-H samples with MLH1 silencing data available are shown. $\mathbf{b}$ Model representing accumulation of clonal and subclonal mutations based on the order of POLE and MSI events. c Mutational spectra of the five cases displaying significant enrichment of signature $\mathrm{E}(P O L E$-exo*) mutations in the clonal subset of mutations, suggesting that the POLE-exo* event preceded acquisition of MSI in these samples. Stacked bars represent the relative contributions of signatures $\mathrm{E}$ and $\mathrm{C}$ to the clonal and subclonal spectra of each patient. In each case, signature $\mathrm{E}$ (blue) is enriched in the clonal spectra 
contributed by POLD1 during lagging-strand synthesis, a hypothesis also proposed by a concurrent study ${ }^{25}$.

Order of repair-deficiency events. We next sought to understand the order in which loss of MMR function and loss of POLE/ $P O L D 1$ proofreading function occurred in tumors. It is possible that loss of POLE/POLD1 proofreading could result in a mutation in an MMR gene leading to loss of MMR pathway function, or conversely, that MSI could result in a POLE/POLD1 exonuclease domain mutation. The majority of cases of sporadic MSI are due to $M L H 1$ promoter methylation, ${ }^{3}$ and if MMR loss precedes a POLE/POLD1-exo mutation, then we may expect a similar proportion of MLH1 methylation in POLE WT vs. POLE-exo* MSI samples. However, the percentage of MSI patients with MLH1 silencing falls from greater than $80 \%$ in POLE-WT cases to less than $50 \%$ in cases with POLE-exo* (Fig. 6a, Fisher's exact $p=$ $2.8 \times 10^{-3}$ ), providing evidence against an exclusively MSI-first model.

We next considered the clonality of the mutations generated in POLE-MSI samples. While the alterations in the MMR or POLE genes in these tumors are typically clonal (Supplementary Figure 1), we reasoned that the earlier of the two events may contribute clonal mutations from its respective signature (E1-3 from a POLE-exo* event or M1-2 from an MSI event) prior to the occurrence of the second event (after which mutations from the signatures of concurrent POLE and MSI loss [C1 and C2] will accumulate, Fig. 6b). Similar approaches were applied to an aggregated cohort in order to infer whether signatures were active early or late in tumor evolution, ${ }^{14,26}$ and a similar analysis of a bMMRD POLE-exo* case by a concurrent study found an enrichment of COSMIC signature 14 in the subclonal mutations of the tumor ${ }^{9}$. Therefore, we searched for enrichment of signature E (POLE-MSS) or M (MSI) among the clonal mutations of 19 cases with substantial contributions (at least 100 mutations and at least $35 \%$ of total) of signatures $\mathrm{C} 1$ and $\mathrm{C} 2$. Using this approach, we were able to identify five (of the 19) samples in which signatures E1-3 were significantly (Methods) overrepresented among the clonal mutations, consistent with a POLE mutation preceding the acquisition of MSI (Fig. 6c, Supplementary Figure 14 and Supplementary Data 7). In these cases, when mutations were separated into clonal and subclonal populations (Methods section), the characteristic signature E peaks, $\mathrm{T}(\mathrm{C}>\mathrm{A}) \mathrm{T}$ and $\mathrm{T}(\mathrm{C}>\mathrm{T}) \mathrm{G}$, were overrepresented among the clonal mutations whereas subclonal mutations more closely resembled the spectrum of signatures $\mathrm{C} 1-2$, suggesting that the POLE mutation occurred prior to loss of mismatch repair. Surprisingly, we noted that $3 / 5$ of these cases displayed MLH1 silencing as the likely mechanism of mismatch repair deficiency, suggesting that even when POLE-exo* precedes acquisition of MSI, loss of MMR function is not always due to POLE-mediated mutagenesis of an MMR gene. Rather, dysregulation of methylation pathways or positive selection of MMR-deficient clones due to the POLE phenotype (e.g., if leaving POLE-induced mismatches unrepaired allows faster completion of S phase) may drive MSI acquisition in these cases.

In the remaining $13 / 19$ cases, we could not identify a clear enrichment of signature $\mathrm{E}$ or $\mathrm{M}$ among the clonal mutations. In cases where the POLE-exo* and MSI events occur in close succession, very few signature $\mathrm{E}$ or $\mathrm{M}$ mutations would accumulate before the second event, and thus identification by this method would be poorly powered. Notably, we were not able to identify cases with significant enrichment of clonal signature $M$ mutations (which would be expected if MSI arose first). However, the mutation rate of signature $\mathrm{C}$ is dramatically higher than that of signature $\mathrm{M}$, and their preferred contexts are largely overlapping; therefore, our power to detect the presence of clonal signature $\mathrm{M}$ mutations is limited.

\section{Discussion}

Cells are exposed to numerous mutagenic processes across their lifetime. Replication errors are an important source of point mutations across the genome, and replication fidelity is typically enforced through dual activity of polymerase proofreading and the MMR pathway. Loss of either of these functions in isolation is associated with increased mutation burden, characteristic mutational signatures, and unique clinical properties. Here, we characterize three mutational signatures that operate in the context of concurrent loss of POLE/POLD1 proofreading and MMR function. These signatures are closely related to COSMIC signatures 14 and 20, and our data provide a mechanistic basis for the activity of these signatures across several tumor types. Given the increasing role of tumor DNA repair deficiency as a predictive clinical biomarker-including the role of MSI and POLE/POLDI mutations as predictors of response to immune checkpoint blockade ${ }^{27-29}$ - a deeper understanding of the mutational processes in these tumors may ultimately inform clinical decisionmaking.

Importantly, the POLE-MSI and POLD1-MSI signatures characterized here do not simply represent the aggregate sum of the two processes, but rather have unique profiles that reflect the biological interaction of POLE/POLD1- and MMR-mediated DNA repair. Our observations demonstrate that signatures can exhibit non-additive properties that are not described by the current paradigm for understanding and modeling mutational processes, and therefore may serve as the basis for new methodologies to discover, analyze, and interpret mutational signatures in cancer. Such improved modeling of DNA damage and repair processes will become increasingly important as more cancer mutation data accumulate.

\section{Methods}

Calling of somatic mutations. In order to assemble the mutation calls for our principal cohort of endometrial samples, alignments (BAM files) of the 547 TCGA exomes were downloaded from the Genome Data Commons (GDC). Somatic point mutations were called using Mutect ${ }^{30}$ and filtered using D-ToxoG ${ }^{31}$, and indels were called using indelocator ${ }^{32}$ (all available at http://www.broadinstitute.org/ cancer/cga). All calls were filtered using a panel of normals. Indels in microsatellite loci were called using MSMutect ${ }^{13}$.

Identification of repair defects. To identify patients with $P O L E$-exo* and POLD1exo $^{*}$, we searched for mutations in the associated domain using mutation calls before panel-of-normal filtering. $P O L E$-exo ${ }^{*}$ and $P O L D 1-$ exo $^{*}$ patients were identified as those with a missense mutation called within the exonuclease domain of the polymerase (codons $268-471$ and $304-517$, respectively ${ }^{12}$ ). All mutations falling outside this interval, mutations affecting splice sites, or leading to loss of function were discounted. Microsatellite instability status, as defined by Bethesda Protocol classification, ${ }^{11}$ and $M L H 1$ silencing was determined as pre-

viously described ${ }^{33}$ from DNA methylation array data publicly available from the GDC.

Estimation of cancer cell fraction. By leveraging copy number and mutation data, ABSOLUTE $^{34}$ is able to estimate the purity and ploidy of samples. Based on this information, it is able to provide the cancer cell fraction (CCF) of each mutation, as well as a confidence interval for the estimate. Sixteen samples for which these measurements could not be determined (due to low purity, low mutation counts, or lack of discrete copy number alterations) were excluded from further analysis, leaving the 531 endometrial samples that constituted our analysis set.

Signature extraction in TCGA UCEC samples. One of the significant challenges of de novo signature extraction in a cohort with heterogeneous mutation burdens is the increased weight of hyper- or ultra-mutant samples on the discovered signatures, which is particularly pressing in the UCEC cohort with many ultra- or hyper-mutant samples. To minimize this effect in our analysis, SignatureAnalyzer was modified in two aspects to accommodate the large mutation burden of POLE and MSI samples. First, we used a penta-nucleotide sequence context for singlenucleotide variants (SNVs) by considering extra contexts 
at the -2 and +2 position around the mutated base, which allows 1536 channels (six possible substitutions, and 256 possible pentamer contexts centered on a given base) compared to 96 channels in the conventional trinucleotide sequence context. The use of an increased number of channels (1536 vs. 96 channels) with additional information from the pentamer context enables a better separation of mutational signatures with similar spectra when considered in 96 channels (such as signatures E1 and E3, which correspond to two different POLE hotspot mutations). Second, to increase discrimination power for MSI samples, which are enriched in short indels, we included insertions and deletions as additional features, along with SNVs, in the signature extraction. According to the size of inserted and deleted bases, we added eight additional channels: INS1, INS2, INS3, and INS $\geq 4$ for insertions and DEL1, DEL2, DEL3, and DEL $\geq 4$ for deletions. Here, INS1-3 (DEL1-3) denote insertions (deletions) with the size of one, two, and three bases, and INS $\geq 4$ (DEL $\geq 4$ ) represents insertions (deletions) with more than three bases. In summary, the input for the signature extraction is a mutation count matrix, where each column, representing a single patient, comprises (1) $1536 \mathrm{SNV}$ channels corresponding to six possible substitutions $(C>A, C>G, C>T, T>A, T>C$, and $T>G)$ and 256 possible pentamer contexts centered at cytosine or thymine and (2) four insertion and four deletion channels corresponding to sizes of one, two, three, and more than three nucleotides.

This matrix was then subjected to the Bayesian non-negative matrix factorization as previously described ${ }^{14,15}$. Out of six independent BayesNMF runs, four converged to the 12-signature solution, while two converged to the 11signature solution. Since the only difference of the 12-signature solution from the 11-signature solution was a split of the APOBEC signature (signature O3) from the broad-spectrum signature (signature $\mathrm{O} 2$ ), we chose the 12-signature solution in downstream analysis.

Signature extraction. in the combined cohort of TCGA UCEC + bMMRD samples Mutation calls for ten bMMRD samples from Shlien et al. ${ }^{8}$ were downloaded from the Nature Genetics journal website. Since the downloaded MAF had only nonsynonymous SNVs, we also considered only non-synonymous SNVs in the 59 samples with signatures of POLE, POLE-MSI, and POLD1-MSI in UCEC cohort (Supplementary Data 6) and combined them with the mutations in the ten bMMRD samples. We again considered the 5-sequence contexts (1536 channels) as was done for the TCGA endometrial cohort, yielding a matrix of 1536-by-69 mutation counts. Out of SignatureAnalyzer's ten independent Bayesian NMF runs, nine converged to the 8-signature solution and one converged to the 9-signature solution. We have chosen the 8-signature solution for downstream analyses.

Comparison of signatures. In order to compare signatures, we projected them into 96-channel space by removing indel contributions and collapsing pentamer channels with a common trimer motif (e.g., the values of the 16 channels AC[C$>\mathrm{T}] \mathrm{GA}, \mathrm{CC}[\mathrm{C}->\mathrm{T}] \mathrm{GA}, \ldots \mathrm{TC}[\mathrm{C}->\mathrm{T}] \mathrm{GT}$ are all summed to calculate the corresponding 96-space channel $\mathrm{C}[\mathrm{C}->\mathrm{T}] \mathrm{G})$.

Signatures were then compared by calculating the cosine similarity between the 96-dimensional vectors. When comparing signatures with multiple components, we solved for coefficients that would maximize the cosine similarity of a linear combination of the components. For example, to compare signature $\mathrm{C}$ (made up of $\mathrm{C} 1$ and $\mathrm{C} 2$ ) to COSMIC signature $14\left(\mathrm{~S}_{14}\right)$, we find values for $\alpha_{1}$ and $\alpha_{2}$ that maximize the quantity:

$$
\frac{\left(\alpha_{1} \mathbf{C} \mathbf{1}+\alpha_{2} \mathbf{C} 2\right) \cdot \mathbf{S}_{14}}{\left\|\alpha_{1} \mathbf{C} \mathbf{1}+\alpha_{2} \mathbf{C} 2\right\|\left\|\mathbf{S}_{14}\right\|}
$$

Estimate of FDR when comparing signatures to COSMIC. In order to estimate the probability of seeing a cosine similarity of 0.99 or higher to a COSMIC signature when fitting a linear combination of two signatures, as observed between signatures $\mathrm{C} 1$ and $\mathrm{C} 2$ with COSMIC signature 14, we employed two approaches. First, for each pair of our 12 signatures, we calculate the cosine similarity with the best COSMIC signature, excluding true-positive matches listed in Supplementary Data 4. For example, signature $\mathrm{O} 1$ is the typical $\mathrm{CpG}$ signature and is expected, in combination with any other signature, to match to COSMIC 1 with high similarity. Therefore, COSMIC 1 is excluded in comparison with signature O1 paired with any other signature. The distribution of similarities of all resulting matches is plotted in Supplementary Figure 7a. Second, to estimate the false positive rate due to mathematical overfitting without the need for identification of true-positive matches, we employed a permutation-based approach. For each signature, we shuffle the contributions of the 96 trinucleotide channels resulting in randomized signatures with equivalent sparsity to our own. We then calculate the cosine similarity to the best match in the COSMIC database. This process is repeated for 10,000 iterations to estimate the distribution of similarities of randomized signatures to the best COSMIC database match. This distribution is shown in Supplementary Figure $7 \mathrm{~b}$.

Calculation of replication timing profiles. For each combination of repair defects (POLE-MSS, POLE-MSI, etc.), we identified samples with overall spectra dominated by the corresponding signature based on the following criteria listed in
Supplementary Data 6. The coding genome was split into four bins using replication timing data from six lymphoblast cell lines ${ }^{22}$. For each bin, we then counted the number of substitutions of each type that occurred in a given sample. This was then compared to the expected mutation count, calculated for a given patient $p$ and substitution $S$ as:

$$
n_{p, S}^{\exp }=\sum_{c \in S} \frac{\lambda_{c, p}}{N_{c}^{\text {global }}} N_{c}^{\text {bin }}
$$

where $c$ is a channel of substitution type $S$ (e.g., for substitution $\mathrm{C}>\mathrm{A}$, channels AA $[\mathrm{C}>\mathrm{A}] \mathrm{AA}, \mathrm{AA}[\mathrm{C}>\mathrm{A}] \mathrm{AC}, \ldots \mathrm{TT}[\mathrm{C}>\mathrm{A}] \mathrm{TT}) . N_{c}$ represents the number of opportunities for a mutation of channel $c$ across the genome (global) or in a given bin (bin), and $\lambda_{c, p}$ represents the mutation burden of channel $c$ for patient $p$, approximated by:

$$
\lambda_{c, p}=\sum_{i=1}^{\mathrm{nsig}} w_{c, i} h_{i, p}
$$

where $w$ and $h$ are elements of the $\mathrm{W}$ and $\mathrm{H}$ matrix from the NMF extraction.

Error bars were estimated by sampling patients with replacement

1000 times, and calculating the standard deviation of the above metric across iterations.

Calculation of replicative strand asymmetries. Patients were selected as in the replication timing analysis. Then, mutational densities were calculated with respect to the leading strand template, as in our previous work ${ }^{24}$, for all 12 possible stranded base substitutions. Asymmetries were then calculated as the $\log _{2}$ ratio of complementary substitutions. Error bars were estimated by sampling mutations with replacement 1000 times and calculating the standard deviation of each measurement.

Test for POLE and MMR deficiency event ordering. To test the ordering of $P O L E-$ exo $^{*}$ and MMR deficiency events, we reasoned that after the first event, mutations of the corresponding signature (E or $\mathrm{M}$ ) would be accumulated before the second event, after which signature $\mathrm{C}$ would accrue. Assuming both events were clonal, this would lead to an enrichment of signature E/M among the clonal mutations, and contributions should be diminished in the subclonal subset. On the other hand, if the events occurred more or less simultaneously, we should see no such enrichment, leading to the following null $\left(H_{0}\right)$ and alternative $\left(H_{\mathrm{a}}\right)$ hypotheses:

$$
\begin{array}{cc}
H_{0}: \frac{\lambda_{E}^{c}}{\lambda_{C}^{c}} \leq \frac{\lambda_{E}^{s}}{\lambda_{C}^{s}} & H_{a}: \frac{\lambda_{E}^{c}}{\lambda_{C}^{c}}>\frac{\lambda_{E}^{s}}{\lambda_{C}^{s}} \\
H_{0}: \frac{\lambda_{M}^{c}}{\lambda_{C}^{c}} \leq \frac{\lambda_{M}^{s}}{\lambda_{C}^{s}} & H_{a}: \frac{\lambda_{M}^{c}}{\lambda_{C}^{c}}>\frac{\lambda_{M}^{s}}{\lambda_{C}^{s}}
\end{array}
$$

where $\lambda^{a}{ }_{b}$ represents the expected mutation count of signature $b$ with clonality $a$.

To calculate our test statistics, probabilities of each mutation having been generated by a given process were assigned as in ref. ${ }^{14}$. Then, probabilities were summed for signatures of a given set (e.g., $P(\operatorname{sigM})=P(\operatorname{sigM} 1)+P(\operatorname{sigM} 2))$. For each patient, mutations were subset to clonal (mutations with $95 \%$ confidence cancer cell fraction $>0.75)$ and subclonal $(95 \%$ confidence of CCF $<0.75)$. The number of clonal $\left(n^{\mathrm{c}}\right)$ and subclonal $\left(n^{\mathrm{s}}\right)$ mutations in each subset contributed by signatures $\mathrm{E}, \mathrm{M}$, and $\mathrm{C}$ was estimated by summing their respective probability values.

We then randomly sampled 1,000,000 clonal and subclonal mutation counts, $n^{\mathrm{c}^{*}}$ and $n^{\mathrm{s}^{*}}$, from Poisson distributions parameterized by $n^{\mathrm{c}}$ and $n^{\mathrm{s}}$ for each signature. $p$ values were then established by counting the fraction of iterations in which

$$
\frac{n_{E}^{c^{*}}}{n_{C}^{c^{*}}}<\frac{n_{E}^{s^{*}}}{n_{C}^{s^{*}}}
$$

for signature E and likewise for signature M. Finally, we applied a Benjamini-Hochberg multiple hypothesis testing correction across patients and determined significance with a threshold of $q<0.1$

Code availability. Code for SignatureAnalyzer (v1.1) that we used to perform mutational signature analysis (with default parameters) can be downloaded from www.broadinstitute.org/cancer/cga under an open-source license.

Data availability. DNA sequencing data and methylation data used in this study are available at the Genome Data Commons website (https://gdc.cancer.gov/).

Received: 8 November 2017 Accepted: 26 March 2018 Published online: 01 May 2018 


\section{References}

1. Alexandrov, L. B. et al. Signatures of mutational processes in human cancer. Nature 500, 415-421 (2013).

2. Supek, F. \& Lehner, B. Differential DNA mismatch repair underlies mutation rate variation across the human genome. Nature 521, 81-84 (2015).

3. Cancer Genome Atlas Research N. et al. Integrated genomic characterization of endometrial carcinoma. Nature 497, 67-73 (2013).

4. Cancer Genome Atlas N. Comprehensive molecular characterization of human colon and rectal cancer. Nature 487, 330-337 (2012).

5. Albertson, T. M. et al. DNA polymerase epsilon and delta proofreading suppress discrete mutator and cancer phenotypes in mice. Proc. Natl Acad. Sci. USA 106, 17101-17104 (2009).

6. Herr, A. J., Kennedy, S. R., Knowels, G. M., Schultz, E. M. \& Preston, B. D DNA replication error-induced extinction of diploid yeast. Genetics 196, 677-691 (2014)

7. Williams, L. N., Herr, A. J. \& Preston, B. D. Emergence of DNA polymerase epsilon antimutators that escape error-induced extinction in yeast. Genetics 193, 751-770 (2013)

8. Shlien, A. et al. Combined hereditary and somatic mutations of replication error repair genes result in rapid onset of ultra-hypermutated cancers. Nat. Genet. 47, 257-262 (2015).

9. Campbell, B. B. et al. Comprehensive analysis of hypermutation in human cancer. Cell 171, 1042-1056 (2017).

10. Billingsley, C. C. et al. Polymerase varepsilon (POLE) mutations in endometrial cancer: clinical outcomes and implications for Lynch syndrome testing. Cancer 121, 386-394 (2015).

11. Umar, A. et al. Revised Bethesda Guidelines for hereditary nonpolyposis colorectal cancer (Lynch syndrome) and microsatellite instability. J. Natl Cancer Inst. 96, 261-268 (2004).

12. Church, D. N. et al. DNA polymerase epsilon and delta exonuclease domain mutations in endometrial cancer. Hum. Mol. Genet. 22, 2820-2828 (2013).

13. Maruvka, Y. E. et al. Analysis of somatic microsatellite indels identifies driver events in human tumors. Nat. Biotechnol. 35, 951-959 (2017).

14. Kasar, S. et al. Whole-genome sequencing reveals activation-induced cytidine deaminase signatures during indolent chronic lymphocytic leukaemia evolution. Nat. Commun. 6, 8866 (2015).

15. Kim, J. et al. Somatic ERCC2 mutations are associated with a distinct genomic signature in urothelial tumors. Nat. Genet. 48, 600-606 (2016).

16. Tan, V. Y. \& Fevotte, C. Automatic relevance determination in nonnegative matrix factorization with the beta-divergence. IEEE Trans. Pattern Anal. Mach. Intell. 35, 1592-1605 (2013).

17. Jin, Y. H. et al. The 3'-- 5' exonuclease of DNA polymerase delta can substitute for the 5' flap endonuclease Rad27/Fen1 in processing Okazaki fragments and preventing genome instability. Proc. Natl Acad. Sci. USA 98, 5122-5127 (2001).

18. Shevelev, I. V., Ramadan, K. \& Hubscher, U. The TREX2 3'-- 5' exonuclease physically interacts with DNA polymerase delta and increases its accuracy. ScientificWorldJournal 2, 275-281 (2002).

19. Shinbrot, E. et al. Exonuclease mutations in DNA polymerase epsilon reveal replication strand specific mutation patterns and human origins of replication. Genome Res. 24, 1740-1750 (2014).

20. Palles, C. et al. Germline mutations affecting the proofreading domains of POLE and POLD1 predispose to colorectal adenomas and carcinomas. Nat. Genet. 45, 136-144 (2013).

21. Schmitt, M. W., Matsumoto, Y. \& Loeb, L. A. High fidelity and lesion bypass capability of human DNA polymerase delta. Biochimie 91, 1163-1172 (2009).

22. Koren, A. et al. Differential relationship of DNA replication timing to different forms of human mutation and variation. Am. J. Hum. Genet. 91, 1033-1040 (2012).

23. Lawrence, M. S. et al. Mutational heterogeneity in cancer and the search for new cancer-associated genes. Nature 499, 214-218 (2013).

24. Haradhvala, N. J. et al. Mutational strand asymmetries in cancer genomes reveal mechanisms of DNA damage and repair. Cell 164, 538-549 (2016).

25. Andrianova, M. A., Bazykin, G. A., Nikolaev, S. I. \& Seplyarskiy, V. B. Human mismatch repair system balances mutation rates between strands by removing more mismatches from the lagging strand. Genome Res. 27, 1336-1343 (2017).

26. McGranahan, N. et al. Clonal status of actionable driver events and the timing of mutational processes in cancer evolution. Sci. Transl. Med. 7, $283 \mathrm{ra} 254$ (2015).
27. Johanns, T. M. et al. Immunogenomics of hypermutated glioblastoma: a patient with germline POLE deficiency treated with checkpoint blockade immunotherapy. Cancer Discov. 6, 1230-1236 (2016).

28. Bouffet, E. et al. Immune checkpoint inhibition for hypermutant glioblastoma multiforme resulting from germline biallelic mismatch repair deficiency. $J$. Clin. Oncol. 34, 2206-2211 (2016).

29. Le, D. T. et al. PD-1 blockade in tumors with mismatch-repair deficiency. $N$. Engl. J. Med. 372, 2509-2520 (2015).

30. Cibulskis, K. et al. Sensitive detection of somatic point mutations in impure and heterogeneous cancer samples. Nat. Biotechnol. 31, 213-219 (2013).

31. Costello, M. et al. Discovery and characterization of artifactual mutations in deep coverage targeted capture sequencing data due to oxidative DNA damage during sample preparation. Nucleic Acids Res. 41, e67 (2013).

32. Van der Auwera, G. A. et al. From FastQ data to high confidence variant calls: the genome analysis toolkit best practices pipeline. Curr. Protoc. Bioinformatics 43, 1110 11-11 1033 (2013).

33. Polak, P. et al. A mutational signature reveals alterations underlying deficient homologous recombination repair in breast cancer. Nat. Genet. 49, 1476-1486 (2017).

34. Carter, S. L. et al. Absolute quantification of somatic DNA alterations in human cancer. Nat. Biotechnol. 30, 413-421 (2012).

\section{Acknowledgements}

We thank Hui Shen and Peter Laird for providing epigenetic silencing calls. G.G. was partially funded by the Paul C. Zamecnick, MD, Chair in Oncology at MGH. N.J.H., Y.E M., P.P., and G.G. were partially funded by G.G. start-up funds at MGH. J.K. and G.G. were partially funded by the NIH TCGA Genome Data Analysis Center (U24CA143845) M.S.L. was funded by start-up funds from the Massachusetts General Hospital Cancer Center. K.W.M. was funded by the NCI (1K08CA219504) and the Burroughs Wellcome Fund.

\section{Author contributions}

N.J.H., J.K., Y.E.M., P.P., K.W.M., M.S.L., and G.G. designed the project. N.J.H., J.K., Y.E M., and M.S.L. carried out analyses. D.R., D.L., and I.L. prepared mutation calls and ABSOLUTE results. A.K. analyzed POLD1 protein structure. J.M.H. advised on analysis. N.J.H., J.K., Y.E.M., K.W.M., M.S.L., and G.G. wrote the manuscript. P.P., K.W.M., M.S. L., and G.G. supervised the project.

\section{Additional information}

Supplementary Information accompanies this paper at https://doi.org/10.1038/s41467018-04002-4.

Competing interests: The authors declare no competing interests.

Reprints and permission information is available online at http://npg.nature.com/ reprintsandpermissions/

Publisher's note: Springer Nature remains neutral with regard to jurisdictional claims in published maps and institutional affiliations.

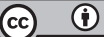

Open Access This article is licensed under a Creative Commons Attribution 4.0 International License, which permits use, sharing, adaptation, distribution and reproduction in any medium or format, as long as you give appropriate credit to the original author(s) and the source, provide a link to the Creative Commons license, and indicate if changes were made. The images or other third party material in this article are included in the article's Creative Commons license, unless indicated otherwise in a credit line to the material. If material is not included in the article's Creative Commons license and your intended use is not permitted by statutory regulation or exceeds the permitted use, you will need to obtain permission directly from the copyright holder. To view a copy of this license, visit http://creativecommons.org/ licenses/by/4.0/.

(c) The Author(s) 2018 